\title{
Measuring optimality of a neural system by a logic gate model
}

\author{
Robert Friedman \\ Retired from Department of Biological Sciences, University of South Carolina, Columbia, SC, USA \\ Correspondence: bob.network.science@gmail.com
}

\begin{abstract}
The nematode worm, Caenorhabditis elegans, is a relatively simple neural system model for measuring the efficiency of information transmission from sensory organ to muscle fiber. With the potential to measure this efficiency, a method is proposed to compare the organization of an idealized neural circuit with a logic gate design. This approach is useful for analysis of a neural circuit that is not tractable to a strictly biological model, and where the assumptions of a logic gate design have applicability. Also, included in the results is an abstract perspective of the electrical-specific synaptic network in the somatic system of the nematode worm.
\end{abstract}

\section{Introduction}

McCulloch and Pitts [1] introduced the concept that a neural system processes information by a biologically plausible logic gate design. However, neural systems are dependent on a biological design that does not necessarily conform to a logic gate model [2-11]. A logic gate is a model for a boolean function, such as the logical operations AND, OR, or NOT. Another model, with an emphasis on biological realism, is based on electrotonic networks [12]. The advantage of a logic gate model and the electrotonic network model, where applicable to a small neural circuit, is explainability. In addition, these models provide a mathematical expression for the events in a hypothetical circuit. They offer a formal hypothesis to help unravel the workings of a neural circuit in animals.

These models most often assume a static environment for an animal, whereas in nature the environment is changing and the neural system is organized for overlap in function. The overlap would remove the requirement for the existence of neural modules for each environmental condition. A non-overlapping system is not an efficient method for a design of an information-based system. At the least, an efficient design would require a large amount of reuse of these local clusters of neurons and synaptic links. However, in the case of a controlled environment and that the neural circuit is simple, it should be possible to map a neural circuit as a set of logic gates.

This question of optimality in a neural system has been examined at the structural and functional level of a network of neurons [8, 12-17]. In the case of Caenorhabditis elegans, one type of optimization is observed in the neuronal wiring length for minimizing the path length to communicate information [14]. Given an organization of cells, it is expected that a neural system is optimized in the cost of development and its operation. One piece of evidence is that an organ system is typically adapated in its physiological and anatomical properties. Another supporting argument is the role of natural selection which will tend to disfavor those individuals in a population that use energy without benefit, such as for development of a larger neural system with a higher energy requirement that offers no advantage over the baseline form. Static properties of the larger neural network in $C$. elegans has also been described, such as the observation of a small world architecture [5, 18-19], sparsity of neuronal connectiveness [11], along with commonly noted motifs that are observed in the network $[3,20]$. 
The simpler neural systems, particularly the neural circuits, are tractable for study and to measure efficiency across the life history of an animal. Past studies have examined the coding of animal behavior by the neural circuits of a system [4, 7, 9, 16, 21-24]. It is further possible to relate these neural systems to the communication of information in animals $[5,25]$. The concept of information flow, efficiency, and the presence of neural circuits suggest a design that shows analogy with logic gates and their boolean functions [3, 26]. The application of engineered designs to a neural system has also been put forward by others, such as Yan and others [16], Karbowski [11], and Lysiak and Paszkiel [27]. In particular, Yan and others [16] proposed a model based on control theory to explain and predict the structure of a neural circuit for locomotion in C. elegans. Their predictions were supported by experiments in C. elegans.

For understanding individual neuron behavior and dynamics in small clusters of neurons, a biologically justified model is an ideal choice. In the case of a larger network of neurons, and in unraveling a neural circuit, where the scale of interest is in the information flow and the corresponding theory, then it is of interest to test with a model of few assumptions with predictiveness on the network efficiency and flow. This approach is the rationale for use of this logic gate model, even though it models by abstraction the lower scale processes at the neuronal level. It also provides a formal procedure for developing hypotheses on the functioning of a neural circuit. The division of perspective at the small and large scale is also seen in the science of ecological systems, another non-linear dynamic system, where the living organism is abstracted away in favor of measurements of the efficiency and flow of energy and matter [28].

\section{Methods}

\section{Data retrieval}

Neuronal data obtained is from the adult hermaphroditic form of Caenorhabditis elegans which has two distinct nervous systems, a somatic system of 282 neurons [29] and a pharyngeal system of 20 neurons [30]. The neuronal connectivity data includes all nonpharygeal neurons except two neurons without synaptic data, CANL and CANR (WormAtlas.Org) [29, 31-34]. The data files were accessed by a web based interface at WormAtlas.Org.

WormAtlas.Org documents the numbers of sensory and motor neuronal types in the adult hermaphroditic form of $C$. elegans. Several of these neurons are not fully characterized along with a set of neurons that are not easily assigned to one of these types, so the following assignments were made based upon the curated databases at WormAtlas.Org. Sensory: ADEL, CEPVL, CEPVR, IL1DL, IL1DR, IL1L, IL1R, IL1VL, IL1VR. Motor: URADL, URADR, URAVL, URAVR. Therefore, of the 282 somatic system neurons, 82 were assigned as a sensory type, 118 as a motor type, and the 82 remaining as interneurons. The neural circuit for gentle touch for locomotion is described by Yan and others [16] and Driscoll and Kaplan [21]. This study also assumes that any neural circuit operates by a forward feedback loop, a biologically reasonable assumption [20].

\section{Data processing and visualization}

Minitab statistical software [35], version 14, was used for data organization and processing. The software Gephi version 0.9.2 was used to construct the graphical networks of neurons, generate random networks, and calculate the frequency distribution of numbers of degrees per node [36].

The diagram of the digital circuit was drawn in TinyCAD [37]. It contains software libraries named 74TTL and gen_Logic which have graphical symbols for many of the common types of logic gates. 
Logic gate analysis

The Espresso software was used to calculate the optimal number of logic gates given a number of inputs and outputs in a digital circuit [38]. Below is an example of input data for use by this software and it corresponds to the AND/NOT case (table 1) with 3 inputs and 4 outputs:
.i 3
.04
.ilb A B C
.ob E F G H
0000000
1000000
0100000
0010000
1101100
1010000
0110011
1110000
. $\mathrm{e}$

The above data contains the formatted truth table where each of the lines of input $(\mathrm{A}, \mathrm{B}, \mathrm{C})$ and output (E, $\mathrm{F}, \mathrm{G}, \mathrm{H})$ values are separated by a space. The header information includes the count of input and output types along with a variable name for each of the types. The software resolves the truth table into a set of logic gates represented by boolean algebraic form. The result for the above data is:

$$
\begin{aligned}
& E=(A \& B \& ! C) \\
& F=(A \& B \& ! C) \\
& G=(! A \& B \& C) \\
& H=(! A \& B \& C)
\end{aligned}
$$

Espresso was run at the command line with parameters to print a solution in the above format: espresso.exe -oeqntott -Dso -S1 -t input_datafile. For a larger sets of inputs and outputs, the above truth table can be duplicated which corresponds to an assumption that each of the duplicated and original circuits are independent of each other (a binary state in one circuit will not affect a state in another circuit). For input values greater than 12 , the time complexity is exponential and so these can be estimated by the corresponding output value. This trend and its estimation is possible given the assumption of independence among the duplicated circuits.

All possible input state values of a truth table may be obtained by enumeration and binary conversion. An example of this method is shown below in bash shell code for the case of 12 inputs and 16 outputs $\left(2^{12}\right.$ combinations of input states):

for i in \$(seq 0 4095); do echo "obase=2;\$i" | bc | tr -d 'r' | xargs printf "\%012d ln"; done 
Table 1. Truth table for a digital circuit with three input and four output operations. Each row corresponds to one of the eight possible input and resultant output states. The binary digit 1 is an ON state and 0 is an OFF state. An OR operation resolves to an $\mathrm{ON}$ state if either of two specific inputs are $\mathrm{ON}$, while an AND operation resolves to an ON state where two specific inputs are ON. NOT modifies the state from ON to OFF or OFF to ON.

\begin{tabular}{|c|c|c|c|}
\hline \multicolumn{2}{|c|}{$\mathrm{OR} / \mathrm{NOT}$} & \multicolumn{2}{|c|}{ AND / NOT } \\
\hline Inputs & Outputs & Inputs & Outputs \\
\hline 000 & 0000 & 000 & 0000 \\
\hline 100 & 1100 & 100 & 0000 \\
\hline 010 & 0000 & 010 & 0000 \\
\hline 001 & 0011 & 001 & 0000 \\
\hline 110 & 1100 & 110 & 1100 \\
\hline 101 & 0000 & 101 & 0000 \\
\hline 011 & 0011 & 011 & 0011 \\
\hline 111 & 00000 & 111 & 0000 \\
\hline
\end{tabular}

\section{Results}

The network of neuronal connections in C. elegans

Fig. 1 shows the network topology of the electrical connections (gap junctions) between neurons of the somatic nervous system of $C$. elegans. The shape and distribution of connections among neurons in this figure is also representative of the connections formed by a chemical synapse [5, 16]. For either synapse type, the network reflects a small world architecture of many distinct clusters of neuronal cells (nodes) and their interconnections (edges). This architecture is also consistent with a modularized structure of the nervous system $[15,19]$.

The data set of all synapses includes 280 neurons of the somatic system and 4577 connections. A random network of this data is expected to average 16.3 connections, but the shape of the distribution is different between the neuronal data and the random network. The latter has a bell-shaped frequency distribution of number of connections per node, while the neuronal data has a distribution where the typical neuron has fewer than 16.3 connections, and a smaller proportion of neurons that are highly connected. These highly connected neurons range from 50 to 200 in number of interconnections. These high values are not expected in a random network.

Of the 280 neurons in the somatic system, 253 neurons are specific to the network of electrical synapses (fig. 1). With the estimate of 1031 gap junctions across this system, there is an expectation of approximately four of these connections per neuron. The relationship between the properties of the neuronal and random network is consistent with that of the entire network of both chemical and electrical synaptic connections ( $\mathrm{N}=4577)$. 


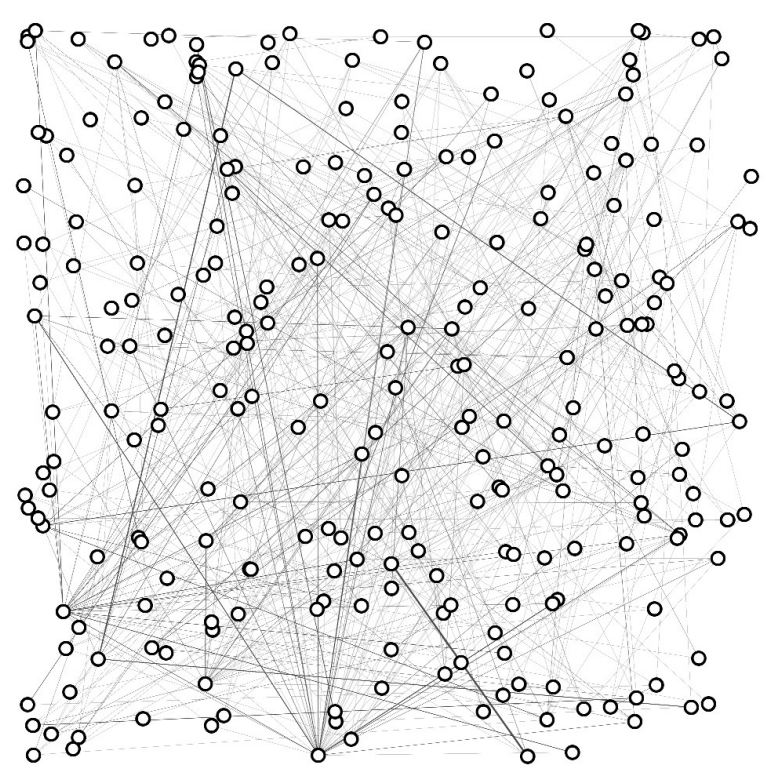

Fig. 1. The network of gap junction connections (grey lines; N=1031) among neurons (open circles; $N=253$ ) of the somatic system in the nematode worm. The line thickness corresponds to the number of gap junction connections between any two connected neurons.

\section{Optimality of an idealized neural circuit}

Yan and others [16] examined a neural circuit in C. elegans of sensory neurons involved in gentle touch, interneurons for control, and motor neurons that synapse with muscle by neuromuscular junctions [21]. They isolated the members of this circuit that are predicted by control theory as crucial to its operation. The inputs include three sensory neurons while the outputs correspond to four motor neurons that synapse on locomotory muscles responsible for forward or backward motion. Interneurons may act as intermediaries for transmission of information between the sensory and motor neurons.

In this study, the logic gate design was determined given the number of sensory inputs and motor outputs. Table 1 shows this data as represented by truth tables and posits two models of a hypothetical, but potentially plausible, neural circuit in animals (fig. 2). This scheme is analogous to the circuit used in C. elegans for gentle touch [16]. Each 1 or 0 in the truth table codes for an on or off state of a neuron in the circuit. A logic gate control scheme is fit to the inputs and outputs by use of the Espresso software [38], and a design is reported that reflects these models (fig. 2). The OR/NOT model allows for multiple neurons to independently code for forward or backward locomotion, while the AND/NOT model requires a neuron to depend on another for any locomotion to occur.

This method is dependent on the validity of a logic gate model as representative of the flow of information from sensory organ to locomotory muscle, and that the information flows in one direction from sensory input to motor output. 


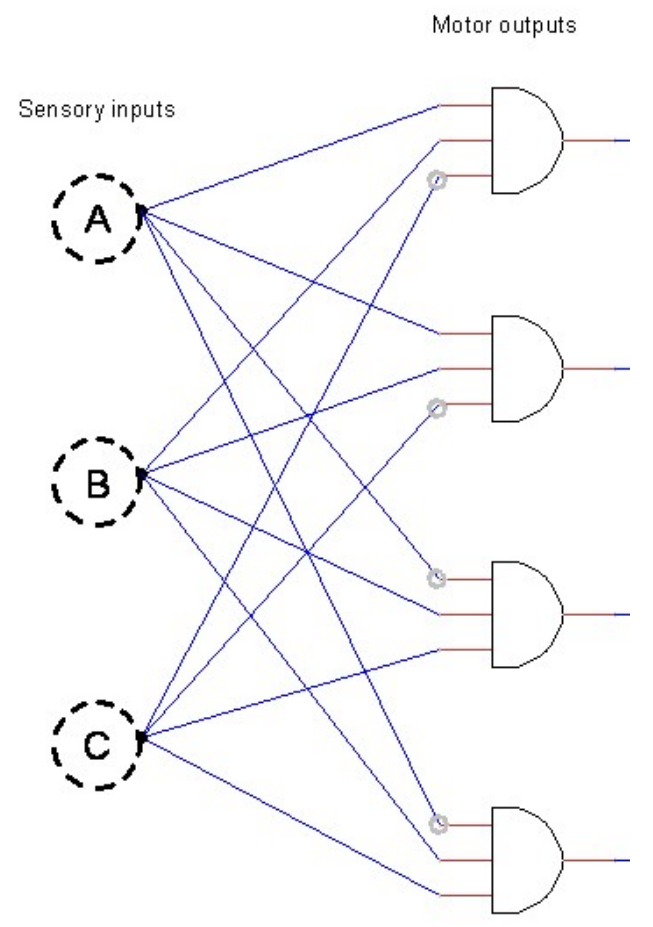

Fig. 2. Diagram of a neural circuit with three sensory inputs and four logic gates that correspond to four motor outputs. The small grey circles represent a potential inverting of the input signal (NOT operator).

\section{Discussion}

Figure 1 supports prior findings about the structure of the $C$. elegans somatic neuronal network. There is a small world architecture with a significant amount of local clustering in the network. The often used analogy for this distribution is the system of world airports and the airplane flights that connect them. In this case, a small proportion of the airports serve as centers, or hubs, where the flights connect to many of the neighboring airports. This analogy and the case of the animal neural network support constraints on the design of the network and the modularity in its organization and structure. This effect may also be considered as an efficient design, given that the majority of the information flow in a neural system is local.

Figure 2 shows the other result of this study, where a model of logic gates is used to explain an idealized neural circuit. This requires an applicability of the model to a problem and the assumptions with abstracting away the dynamics at the neuronal level [25]. In a large network, and for higher information processing, it is expected that other approaches are applicable, such as the use of non-linear dynamics for capturing the deeper complexity in a neural system, and by the use of comparisons between animal and artificial neural networks [39-40]. However, at these larger scales, there is typically a limitation on the explainability of a model at the neuronal and synaptic level. Use of a logic gate model may provide a guide on the flow and efficiency of information in a neural circuit, given the assumptions of the model to a circuit [25].

Another assumption of this model is that the environment is unchanging. If the environment is dynamic, and there is no control on its potential for change, then it is difficult to disentangle a neural circuit. It is a reminder that past studies have often identified roles of neurons in simple neural systems, but these roles are particular to an environment or a set of environments, notwithstanding the changes an animal experiences from other causes. Another way to express this complex problem is by describing it as a high dimensional system, 
and a reason to use other approaches for understanding a neural system at this large scale perspective, such as the use of abstract patterns in a network of neurons.

Apart from the applicability of this model, it is also a concept that information is flowing from sensory input to output, such as in the case of sensory to motor neuron [41]. The neural network and the circuits are expected to form based on the occurrence of inputs and outputs, especially in the formation of a neural system over an animal's development. It is in essence a programmable system of neurons and synaptic links that connect the neurons. These links, at least as idealized features, are dynamic with respect to gain, loss, and strength of connection. The logic gate model, as described in an above section, does not capture these parameters of a dynamic system, but it does offer a static view of a neural system and the roles of neurons in a controlled environment. This is a view that does not exclude other sources of variability in the system. The logic gate model would serve as a tool, a null hypothesis, for helping to explain the optimality of a neural circuit.

\section{Ethics approval and consent to participate}

Not applicable.

\section{References}

[1] McCulloch WS, Pitts W. 1943. A logical calculus of the ideas immanent in nervous activity. The Bulletin of Mathematical Biophysics 5: 115-133.

[2] Smith M, Pereda AE. 2003. Chemical synaptic activity modulates nearby electrical synapses. Proceedings of the National Academy of Sciences 100: 4849-4854.

[3] Reigl M, Alon U, Chklovskii DB. 2004. Search for computational modules in the C. elegans brain. BMC Biology 2: 25.

[4] Gray JM, Hill JJ, Bargmann CI. 2005. A circuit for navigation in Caenorhabditis elegans. Proceedings of the National Academy of Sciences of the United States of America 102: 3184-3191.

[5] Varshney LR, Chen BL, Paniagua E, Hall DH, Chklovskii DB. 2011. Structural properties of the C. elegans neuronal network. PLOS Computational Biology 7: e1001066.

[6] Bargmann CI. 2012. Beyond the connectome: how neuromodulators shape neural circuits. Bioessays 34: 458-465.

[7] Zhen M, Samuel AD. 2015. C. elegans locomotion: small circuits, complex functions. Current Opinion in Neurobiology 33: 117-126.

[8] Niven JE, Chittka L. 2016. Evolving understanding of nervous system evolution. Current Biology 26: R937-R941.

[9] Rakowski F, Karbowski J. 2017. Optimal synaptic signaling connectome for locomotory behavior in Caenorhabditis elegans: Design minimizing energy cost. PLOS Computational Biology 13: e1005834.

[10] Jabeen S, Thirumalai V. 2018. The interplay between electrical and chemical synaptogenesis. Journal of Neurophysiology 120: 1914-1922.

[11] Karbowski J. 2019. Deciphering neural circuits for Caenorhabditis elegans behavior by computations and perturbations to genome and connectome. Current Opinion in Systems Biology 13: 44-51.

[12] Niebur E, Erdos P. 1993. Theory of the locomotion of nematodes: Control of the somatic motor neurons by interneurons. Mathematical Biosciences 118: 51-82.

[13] Laughlin SB, Sejnowski TJ. 2003. Communication in neuronal networks. Science 301: 1870-1874.

[14] Chen BL, Hall DH, Chklovskii DB. 2006. Wiring optimization can relate neuronal structure and function. Proceedings of the National Academy of Sciences 103: 4723-4728. 
[15] Bullmore E, Sporns O. 2012. The economy of brain network organization. Nature Reviews Neuroscience 13: 336-349.

[16] Yan G, Vertes PE, Towlson EK, Chew YL, Walker DS, Schafer WR, Barabasi AL. 2017. Network control principles predict neuron function in the Caenorhabditis elegans connectome. Nature 550: 519-523.

[17] Poznanski RR. 2002. Dendritic integration in a recurrent network. Journal of Integrative Neuroscience 1: 69-99.

[18] Watts DJ, Strogatz SH. 1998. Collective dynamics of 'small-world' networks. Nature 393: 440-442.

[19] Towlson EK, Vertes PE, Ahnert SE, Schafer WR, Bullmore ET. 2013. The rich club of the C. elegans neuronal connectome. The Journal of Neuroscience 33: 6380-6387.

[20] Milo R, Shen-Orr S, Itzkovitz S, Kashtan N, Chklovskii D, Alon U. 2002. Network motifs: simple building blocks of complex networks. Science 298: 824-827.

[21] Driscoll M, Kaplan J. 1997. Mechanotransduction, in The Nematode C. elegans, II (Cold Spring Harbor Press, Cold Spring Harbor, NY). pp. 645-677.

[22] Wakabayashi T, Kitagawa I, Shingai R. 2004. Neurons regulating the duration of forward locomotion in Caenorhabditis elegans. Neuroscience Research 50: 103-111.

[23] Chatterjee N, Sinha S. 2008. Understanding the mind of a worm: hierarchical network structure underlying nervous system function in C. elegans. Progress in Brain Research 168: 145-153.

[24] Campbell JC, Chin-Sang ID, Bendena WG. 2015. Mechanosensation circuitry in Caenorhabditis elegans: A focus on gentle touch. Peptides 68: 164-174.

[25] Poznanski RR. 2001. Biophysical Neural Networks: Foundations of Integrative Neuroscience (Mary Ann Liebert, New York). pp. 177-214.

[26] Goldental A, Guberman S, Vardi R, Kanter I. 2014. A computational paradigm for dynamic logic-gates in neuronal activity. Frontiers in Computational Neuroscience 8: 52.

[27] Lysiak A, Paszkiel S. 2021. A Method to Obtain Parameters of One-Column Jansen-Rit Model Using Genetic Algorithm and Spectral Characteristics. Applied Sciences 11: 677.

[28] Odum EP. 1968. Energy flow in ecosystems - a historical review. American Zoologist 8: 11-18.

[29] White JG, Southgate E, Thomson JN, Brenner S. 1986. The structure of the nervous system of the nematode Caenorhabditis elegans. Philosophical Transactions of the Royal Society B: Biological Sciences 314: 1-340.

[30] Albertson DG, Thomson JN. 1976. The pharynx of Caenorhabditis elegans. Philosophical Transactions of the Royal Society B: Biological Sciences 275: 299-325.

[31] Durbin RM. 1987. Studies on the Development and Organisation of the Nervous System of Caenorhabditis elegans. PhD thesis. UK: University of Cambridge.

[32] Achacoso TB, Yamamoto WS. 1992. AY's Neuroanatomy of C. elegans for Computation (CRC Press, Boca Raton, FL).

[33] Hall DH, Russell RL. 1991. The posterior nervous system of the nematode Caenorhabditis elegans: serial reconstruction of identified neurons and complete pattern of synaptic interactions. Journal of Neuroscience 11: $1-22$.

[34] Hobert O, Hall DH. 1999. Neuroanatomy: A second look with GFP reporters and some comments. Worm Breeder's Gazette (October issue).

[35] Wild DJ. 2005. MINITAB Release 14. Journal of Chemical Information and Modeling 45: 212.

[36] Bastian M, Heymann S, Jacomy M. 2009. Gephi: an open source software for exploring and manipulating networks. International AAAI Conference on Weblogs and Social Media.

[37] Pyne M. 2016. TinyCAD source code (version 2.90.00). https://sourceforge.net/projects/tinycad/. 
[38] Brayton RK, Hachtel GD, McMullen CT, Sangiovanni-Vincentelli AL. 1985. Logic Minimization Algorithms for VLSI Synthesis (Kluwer Academic, Berlin, Germany).

[39] Alom MZ, Taha TM, Yakopcic C, Westberg S, Sidike P, Nasrin MS, Hasan M, Van Essen BC, Awwal AAS, Asari VK. 2019. A State-of-the-Art Survey on Deep Learning Theory and Architectures. Electronics 8: 292.

[40] Bengio Y, Lecun Y, Hinton G. 2021. Deep learning for AI. Communications of the ACM, 64: 58-65.

[41] Friedman R. 2021. Test of robustness of pharyngeal neural networks in Caenorhabditis elegans. NeuroReport 32: 169-176. 